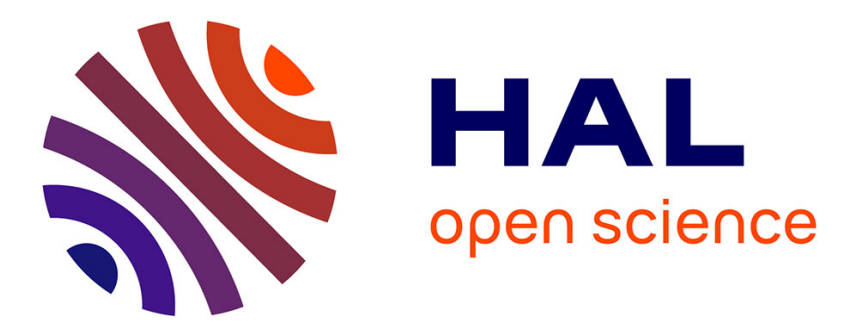

\title{
Survivability in optical networks: a solution for the wavelength continuity constraint case
}

Nicolas Jara, Hermann Pempelfort, Gerardo Rubino, Reinaldo Vallejos

\section{To cite this version:}

Nicolas Jara, Hermann Pempelfort, Gerardo Rubino, Reinaldo Vallejos. Survivability in optical networks: a solution for the wavelength continuity constraint case. LADC 2019 - 9th Latin-American Symposium on Dependable Computing, Nov 2019, Natal, Brazil. pp.1-9. hal-02434311

\section{HAL Id: hal-02434311 \\ https://hal.inria.fr/hal-02434311}

Submitted on 9 Jan 2020

HAL is a multi-disciplinary open access archive for the deposit and dissemination of scientific research documents, whether they are published or not. The documents may come from teaching and research institutions in France or abroad, or from public or private research centers.
L'archive ouverte pluridisciplinaire HAL, est destinée au dépôt et à la diffusion de documents scientifiques de niveau recherche, publiés ou non, émanant des établissements d'enseignement et de recherche français ou étrangers, des laboratoires publics ou privés. 


\section{Survivability in optical networks: a solution for the wavelength continuity constraint case}

\author{
Nicolás Jara \\ Department of Electronics \\ UTFSM \\ Valparaíso, Chile \\ nicolas.jara@usm.cl
}

\author{
Hermann Pempelfort \\ Department of Electronics \\ UTFSM \\ Valparaíso, Chile \\ hermann.pempelfort@usm.cl
}

\author{
Gerardo Rubino \\ INRIA Rennes \\ Bretagne Atlantique \\ Rennes, France \\ gerardo.rubino@inria.fr
}

\author{
Reinaldo Vallejos \\ Department of Electronics \\ UTFSM \\ Valparaíso, Chile \\ reinaldo.vallejos@usm.cl
}

\begin{abstract}
Survivability of internet services is a significant and crucial challenge in designing future optical networks. A robust infrastructure and transmission protocols are needed to handle such a situation so that the network users can maintain communication despite the existence of one or more failed components on the network. For this reason, in this work, we present a generalized approach to tolerate any set of failure scenarios, to the extent the user can still communicate with the remaining components, where a scenario corresponds to an arbitrary set of links in a non-operational state. To assess the survivability problem, we propose a joint solution to the problems listed next. We show how to find: a set of primary routes, a set of alternate routes associated to each failure scenario, and the capacity required on the network to allow communication between all users, in spite of any failure scenario, while satisfying for each user a specific predefined quality of service threshold, defined in the Service Level Agreement (SLA). Numerical results show that the proposed approach not only enjoys the advantages of low complexity and ease of implementation but is also able to achieve significant resource savings compared to existing methods. The savings are higher than $30 \%$ on single link failures and more than a $100 \%$ on two simultaneous link failures scenarios and in more complex failure scenarios.
\end{abstract}

Index Terms-Dynamic WDM Optical Networks, Blocking Probability, Wavelength Continuity, Wavelength Dimensioning, Wavelength Assignment, Routing, Fault Tolerance.

\section{INTRODUCTION}

A remarkable issue to be solved in designing WDM optical networks is to ensure that the network will still be able to provide its transmission service after the failure of one or more of its links. The solution to this problem consists in providing the necessary infrastructure to rapidly re-establish communications between all source-destination pair of nodes affected by these link failures. This type of mechanism is known as "Fault Tolerance".

The frequency of link failures occurrences is significant. For instance, [1], [2] reports measures of the mean time between failures of about 367 year $/ \mathrm{km}$. This failure frequency explains why failures on links may significantly impact the performance of the optical networks. For example, in a 26,000 km-long network such as NSFNet [3], there is an average of one fiber cut every 5 days. Moreover, the frequency with which two simultaneous links failures occur is high enough to be considered in the design process. In fact, Schupke [2] reported that the probability of two simultaneous failures occurring in a network like NSFNet is approximately 0.0027 , implying a downtime of about 24 hours per year, on average, which in addition to the high transmission rate of this kind of networks, means an unacceptable data loss.

The previous elements justify the need to provide an efficient methodology for multiple fault tolerance, which should ensure (with a certain probabilistic guarantee) successful communications among all network users, despite the occurrence of failures in some of the links, and at the lowest possible cost regarding the network infrastructure.

The fault tolerance methods proposed so far generally have been devoted to finding alternative paths considering single link failure (one bidirectional link), affecting all the connections with routes passing through the failed link in both directions. Then, the number of wavelengths in the network is dimensioned to tolerate this situation [2], [4], [5]. However, as already pointed out, the probability of occurrence of two or more simultaneous failures is high enough that it is needed to consider this kind of event in the design of the network. Some studies have focused on this 2-failures scenario [6]-[9].

Also, some studies have considered more complex cases of failures, such as Disaster risk constraints and SharedRisk-Group scenarios. Disaster risk constraints [10]-[12] considers the possible service disruptions in case of a natural disaster or a targeted attack, in which case, the failures affect various links simultaneously. On the other hand, SharedRisk-Group (SRG) [13], [14] considers the possibility that some fibers are placed physically together, even if they are connecting different optical nodes. This scenario makes those fibers liable to physical cuts since they can be cut together at the same time.

Next, we briefly list the most common methods currently used to provide fault tolerance.

One of the most frequent ways used to address simple and double fault tolerance, called " $1+1$ ", can be found in [4], [15], [16]. In this technique, a secondary route is associated with each primary one (with the restriction that they don't share any link), and the information is transmitted simultaneously through both of them. To dimension the number of wavelengths of each link -a task usually done 
by simulation-, each secondary route is considered as just another network route with a load equal to the corresponding primary one. The $1+1$ method is also scalable to provide tolerance to $K \geq 1$ simultaneous failures. In this case, for each connection, $K+1$ supplementary disjoint routes must be found, one as the primary route and the remaining $K$ as secondary routes. Observe that a necessary and sufficient condition that allows this scheme to work is that the graph defined by the set of nodes and links is $(K+1)$-connected.

Another fault tolerance strategy is known as "Shared Path Protection" (SPP) [9], [17]-[19]. In this scheme, the extra resources (wavelengths) assigned to the secondary routes can be shared by different connections, and are assigned only when a fault occurs. The SPP can be executed in two different ways. The first one consists of running the algorithm off-line, where the routes are calculated prior to the operation of the network (off-line SPP). The second way is the on-line implementation (on-line SPP). In this last case, the primary routes are computed before the network is operating, but it must be executed again every time that one or more simultaneous failures occur to compute alternate paths to the affected communications. For this reason, it is said that this is a proactive and reactive approach at the same time.

In [6], [7], [20]-[22] another method of fault tolerance called " $p$-cycle" is discussed, which allows sharing resources through fixed secondary routes that have a cyclic form. These cyclic routes are shared between several primary routes. One problem with this approach is that the applicability of the idea is very dependent on the size of the network, introducing an excessive additional delay for a connection in protection state on large networks. Also, to perform multiple fault tolerance, it requires a large number of cycles (e.g., hundreds of cycles for the 11 nodes pan-European COST 239 network [20]), which is impractical from various points of view.

Here we propose a new fault-tolerance scheme, which we call the "Cheapest Path By Layers with Fault Tolerance" method- CPLFT. In this approach, we go one step further concerning previous works, and we take into account the case of arbitrary sets of links failures scenarios, where a failure scenario is composed by a set of links in failure state. This means that we solve the fault-tolerance problem in its more general aspects.

The method also evaluates the number of wavelengths $W_{\ell}$ for each link $\ell$ of the network, ensuring that the blocking probability of any connection request, of any user $c$, is lower than the corresponding predefined threshold $\beta_{c}$, despite the possible occurrence of those simultaneous link failures. The value of $\beta_{c}$ is defined in the Service Level Agreement (SLA), signed by the service providers and their clients, which defines the minimum quality of service (QoS) acceptable for each user, measured as a probabilistic guarantee. The definition of these bounds is obtained considering objective criteria, such as: different quality of service requirements [23][25]; and subjective decisions, such as network scalability re- quirements. Based on these QoS agreements, engineers must design the network fulfilling said QoS requirements. Thus, we assume that the $\beta_{c}$ values are given and acknowledged by the users and the network service providers.

The remainder of this paper is as follows: In Section II we present the proposed method. Section III contains some results obtained by the proposed algorithm, which are compared with those obtained with the current best methods in a set of different scenarios. Finally, the conclusions of the study are given in Section IV

\section{THE PROPOSED FAULT TOLERANCE METHOD}

\section{A. Model}

The network topology is represented by a graph $\mathcal{G}=$ $(\mathcal{N}, \mathcal{L})$, where $\mathcal{N}$ is the set of network nodes or vertices and $\mathcal{L}$ is the set of unidirectional links (the arcs in $\mathcal{G}$ ), with respective cardinalities $|\mathcal{N}|=N$ and $|\mathcal{L}|=L$. The set of connections $\mathcal{X} \subseteq \mathcal{N}^{2}$, with cardinality $|\mathcal{X}|=X$, is composed by all the source-destination pairs with communication between them. These connections are also called "users" in the text.

We use an ON-OFF model to represent the traffic between a given source-destination pair. Consider connection $c$. During any of its ON periods, whose average length is $t_{O N_{C}}$, the source transmits at a constant rate (which is the rate associated with the wavelength). During an OFF period, with average length $t_{O F F c}$, the source refrains from transmitting data.

The used technology determines the constant transmission rate during the ON periods, but to simplify the presentation, it is our rate unity. Consequently, the traffic load of connection $c$, denoted by $\varrho_{c}$, is given by:

$$
\varrho_{c}=\frac{t_{O N_{c}}}{t_{O N_{c}}+t_{O F F c}} \text {. }
$$

Observe that we address here the general case where the load can be different for each connection, the so-called heterogeneous situation.

Let $\mathcal{R}=\left\{r_{c} \mid c \in \mathcal{X}\right\}$ be the set of routes that enable communications among the different users, where $r_{c}$ is the route associated with connection $c \in \mathcal{X}$. The set $\mathcal{R}$ is known as the set of primary routes since this set alone does not offer any fault tolerance to the possible failure of network links.

Let $\mathcal{W}=\left\{W_{\ell} \mid \ell \in \mathcal{L}\right\}$ be the set containing the number of wavelengths of each unidirectional network link, where $W_{\ell}, \ell \in \mathcal{L}$, is the number of wavelengths on link $\ell$. The value $W_{\ell}$, for every $\ell \in \mathcal{L}$, must be evaluated so that the blocking probability $B P_{c}$ of each connection $c \in \mathcal{X}$ is less than or equal to a given pre-specified threshold $\beta_{c}$, and the total number of available network wavelengths is as small as possible (saving resources).

Remark that the pre-defined threshold value $\beta_{c}$ can be different for each network connection, which means that we treat the general case where there are classes of users with different quality of services (QoS).

As in several works [26]-[28], in this proposal the total network cost $C_{n e t}$ is defined as the sum of all wavelengths 
of all network links, that is, $C_{n e t}=\sum_{\ell \in \mathcal{L}} W_{\ell}$. Because we are considering fault tolerance capabilities, this must include all the additional wavelengths needed to provide tolerance to multiple link failures.

Let $\Omega$ be the set of every possible failure scenarios, where each scenario is a subset $\mathcal{F}$ of failed network links with $\mathcal{F} \subset \mathcal{L}$. The method explained below can be applied to any possible set of failure scenarios. For example, every possible single failure scenario $(|\mathcal{F}|=1)$; every possible double link failure scenario $(|\mathcal{F}|=2)$; a node failure, where all the links connected to that node are considered non-operational; disaster risk constraints [29], [30] where all the links affected by the disaster are considered non-operational; Shared-RiskGroup (SRG) [31] where $\mathcal{F}$ is composed by every link that can be affected by the same physical cut. Note that, all the previous examples consist in only one failure scenario, however the method is designed to offer fault tolerance to any set of scenarios. Remark also that the fault-tolerance guarantee occurs only if the network remains connected after any of the failure scenarios considered, which implies that the method can provide alternative routes for all affected connections.

Some additional definitions required for the explanation of the method are presented in the following list:

\section{B. Definitions and sub-procedures needed by our method}

Since the graph representing the network topology and the set of users are fixed data, as well as the upper bounds $\beta_{c} \forall c \in \mathcal{X}$ of the blocking connection probabilities, we omit them in the list of the parameters of the procedures. $\mathrm{Bu}$ simplicity, when we refer to the network capacity, we write $C_{n e t}$, because we must change the capacities of the links many times during the computational process.

Some definitions required for the explanation of the method are presented in the following list:

- $\mathcal{G}_{-\mathcal{F}}=(\mathcal{N}, \mathcal{L} \backslash \mathcal{F})$, is the partial graph of $\mathcal{G}$ (same nodes, part of the edges), containing only the non-failed links, where $\mathcal{F}$ contains the set of failed links.

- $\mathcal{X}_{\mathcal{F}}=\left\{c \in \mathcal{X} \mid r_{c} \cap \mathcal{F} \neq \phi\right\}$, is the set of connections $c$ affected by the failure $\mathcal{F}$;

- $\mathcal{A}_{\mathcal{F}}=\left\{r_{c} \in \mathcal{R} \mid r_{c} \cap \mathcal{F} \neq \phi\right\}$, is the subset of the routes in $\mathcal{R}$ disables because of the failure $\mathcal{F}$;

- $\mathcal{R}_{\mathcal{F}}$ is a set of routes that replace those in $\mathcal{A}_{\mathcal{F}}$ when all links in $\mathcal{F}$ are failed;

- $\mathcal{S}_{\mathcal{F}}$ is the total set of routes guaranteeing fault tolerance to the failure event "all links in $\mathcal{F}$ fail". That is, the set defined by $\mathcal{S}_{\mathcal{F}}=\left(\mathcal{R} \backslash \mathcal{A}_{\mathcal{F}}\right) \cup \mathcal{R}_{\mathcal{F}}$.

- $\mathcal{C}_{\mathcal{F}}=\left\{\mathcal{C}_{\ell} \mid\right.$ for all $\left.\mathcal{L} \backslash \mathcal{F}\right\}$ is the costs of each link non-affected by the failure $\mathcal{F}$.

The method also needs a few sub-procedures to work. They are described next.

- PrimaryRoutes(). The method starts by computing a set of primary routes. The selection of the route can be made by any available technique, e.g., Dijkstra algorithm [32]. Initially, the method also evaluates the number of wavelengths $W_{\ell}$, for each link $\ell$ of the network, ensuring that the blocking probability of any connection request will be lower than the predefined threshold $\beta_{c}$ (assuming that no failure can happen).

To represent the execution of this sub-procedure, let us symbolically write $\{\mathcal{R}, \mathcal{W}\}:=$ PrimaryRoutes ()

- SecondaryRoutes(). Considering that we have a set of failure links $\mathcal{F}$, the set of costs $\mathcal{C}_{\mathcal{F}}$, and a set of users $\mathcal{X}_{\mathcal{F}}$ affected by the failure of the links in $\mathcal{F}$. We want to find a new set of routes allowing to connect each user in $\mathcal{X}_{\mathcal{F}}$ despite the failure scenario $\mathcal{F}$, while still satisfying the QoS required by each connection.

The search for new routes is done as follows. We run Dijkstra's algorithms looking, for each user $c \in \mathcal{X}$, the cheapest route, where the link costs are now given by the link costs in $\mathcal{C}_{\mathcal{F}}$ (explained later in the algorithm). This procedure creates a new set of routes, that we denote $\mathcal{R}_{\mathcal{F}}$.

Symbolically, we execute this sub-procedure by calling $\mathcal{R}_{\mathcal{F}}:=$ SecondaryRoutes $\left(\mathcal{X}_{\mathcal{F}}, \mathcal{F}, \mathcal{C}_{\mathcal{F}}\right)$.

- Dimensioning(). This procedure consists in finding, for each link $\ell \in \mathcal{L}$, a capacity $W_{\ell}$ such that the endto-end blocking probability $B P_{c}$ of every user $c \in \mathcal{X}$ passing through the link $\ell$ is less than the given threshold $\beta_{c}$. For different reasons, the usual dimensioning procedures consider homogeneity in the links' capacities, that is, look for a capacity $W$, the same on all links, such that the performance objective is reached. We will then follow here the same approach, because this can facilitate further comparisons with existing methods.

The idea is simple: we are given the operational links of the networks, the set of routes $\mathcal{R}$ (because the procedure is used for a diverse set of routes), and the set of quality of service bounds $\beta_{c}$. We then initialize the network capacity $W$ by value 1 and we evaluate the blocking probabilities per user; then, we check if the blocking probability of each user is less than the one defined on the SLA. If the condition is satisfied, we stop the algorithm. If not, we increase $W$ by 1 and we repeat the procedure.

Symbolically we evaluate this sub-procedure by writing: $\{\mathcal{W}\}=\operatorname{Dimensioning}\left(\mathcal{L}, \mathcal{R},\left\{\beta_{c}, \forall c \in \mathcal{X}\right\}\right)$.

\section{The proposed method}

Figure 11 contains a diagram with the inputs required, the condition to be guaranteed, and the outputs obtained by the method execution. The inputs are: the network topology $\mathcal{G}=(\mathcal{N}, \mathcal{L})$, which can be any network topology; each user traffic load $\varrho_{c}$, for all connections in $c \in \mathcal{X}$ (notice that, the value $\varrho_{c}$ of each user $c$ can be different); and the set $\Omega=\{\mathcal{F} \mid \mathcal{F} \subset \mathcal{L}\}$ composed by all the link failure scenarios to be considered by the method execution.

A constraint to be satisfied by the method is to guarantee a given blocking probability $\beta_{c}$ to each network user $c$, predefined on the Service Level Agreement (SLA).

Finally, the method's outputs are the set of primary routes $\mathcal{R}$, allowing to provide communication to each network connection $c$, for all $c \in \mathcal{X}$, under the condition of 


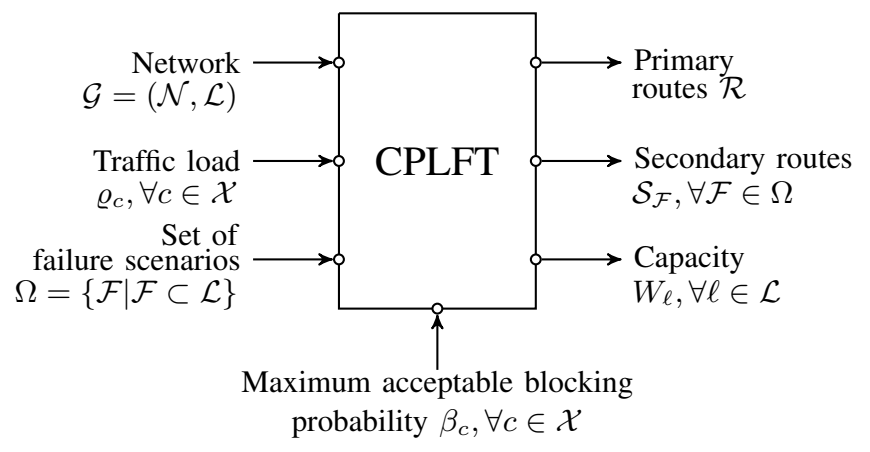

Figure 1. Diagram showing the inputs required to run the CPLFT method and its outputs.

no link failure; the set of alternative routes $\mathcal{S}_{\mathcal{F}}$, for all failure scenarios $\mathcal{F} \in \Omega$, which allow communicating in spite of the links in $\mathcal{F}$ are not operational; and the amount of wavelength $W_{\ell}$ necessary on each network link $\ell$, for all $\ell \in \mathcal{L}$, considering every possible failure scenario in $\Omega$.

We use LIBPE method [33] to compute the connection blocking probabilities necessary to evaluate the quality of service offered to each connection $c$. This procedure is an accurate, fast, and simple technique for the case where exist wavelength continuity constraints. The rate of the blocking probability evaluation execution is significant since, to solve the previously listed problems simultaneously, it is necessary to execute the blocking probability evaluation a lot of times (hundred) considering all failure cases of the set $\Omega$, and in each of these cases to execute the dimensioning procedure. Therefore, it is not possible to evaluate the blocking probability by means of simulation due to the time-consuming task involved.

In algorithmic form, the CPLFT method is presented in Figure 2,

In line $\mathbf{1}$, by using the sub-procedure PrimaryRoutes, we first use Dijkstra algorithm [32] to obtain an initial set of primary routes $\mathcal{R}$, and the set of capacities $\mathcal{W}$ compatible with the QoS constraints $\beta_{c}, c \in \mathcal{X}$, assuming no failures.

Then in line 2, we include the set of every possible failure scenarios $\Omega$, where each of these scenarios is a subset of failed network links $\mathcal{F}$. To explain how the procedure works, initially, assume that the only possible failure scenario is the simultaneous failures of all links in a specific subset $\mathcal{F}$ of $\mathcal{L}$.

In lines 4 to 7, we first start by finding replacement routes in case of the failure of all links in the subset of links $\mathcal{F}$. If a route $r_{c}$ does not use any link of $\mathcal{F}$, it is not changed. However, for all connections $c$ whose route $r_{c} \in \mathcal{R}$ uses at least one link of $\mathcal{F}$ (that is, for all $c \in \mathcal{X}_{\mathcal{F}}$ ), we must find a new route that avoids the links of $\mathcal{F}$. To his end, for every link $\ell \in \mathcal{L} \backslash \mathcal{F}$, we define its $\operatorname{cost} \mathcal{C}_{\ell}$ through the expression:

$$
\mathcal{C}_{\ell}=e^{\varrho_{\ell}-\bar{\varrho}}
$$

where $\varrho_{\ell}$ is the traffic load offered to the link $\ell$ by the connections non-affected by the failed links, and $\bar{\varrho}$ is the mean traffic load evaluated on all the links $\ell$, that is $\ell \mid \ell \in \mathcal{L} \backslash \mathcal{F}$. Then, with these $\mathcal{C}_{\ell}$ values as weights, we run the Dijkstra's algorithm to find the cheapest route for each connection $c \in \mathcal{X}_{\mathcal{F}}$. The set of all these routes are denoted by $\mathcal{R}_{\mathcal{F}}$. Symbolically, we execute the call: $\mathcal{R}_{\mathcal{F}}:=$ SecondaryRoutes $\left(\mathcal{X}_{\mathcal{F}}, \mathcal{F}, \mathcal{C}_{\mathcal{F}}\right)$.

After that, line 8 defines the set of routes $\mathcal{S}_{\mathcal{F}}$ :

$$
\mathcal{S}_{\mathcal{F}}=\left(\mathcal{R} \backslash \mathcal{A}_{\mathcal{F}}\right) \cup \mathcal{R}_{\mathcal{F}}
$$

In words, $\mathcal{S}_{\mathcal{F}}$ is the set of routes to be used when all links in $\mathcal{F}$ are failed. Under this condition, we must dimension the links again because we must always respect the QoS constraints. For this purpose, we restrict the analysis to the graph $\mathcal{G}^{-\mathcal{F}}$, that is, we remove the links in $\mathcal{F}$ from $\mathcal{L}$. Then in line 9, we run a dimensioning phase. In pseudoalgorithmic form, we execute the function call $\mathcal{W}_{\mathcal{F}}:=$ $\operatorname{Dimensioning}\left(\mathcal{L} \backslash \mathcal{F}, \mathcal{S}_{\mathcal{F}},\left\{\beta_{c}, \forall c \in \mathcal{X} \backslash \mathcal{X}_{\mathcal{F}}\right\}\right)$.

Repeating the steps explained above, we obtain a set of secondaries routes for each failure scenario $\mathcal{F}$ of $\Omega$, and the corresponding links dimensioning for each failure scenario.

To finish, in lines 10 to $\mathbf{1 1}$, for every failure scenario $\mathcal{F}$ in $\Omega$, we compare each $W_{\mathcal{F}, \ell}$ (the number of wavelengths of link $\ell$ under each failure scenario $\mathcal{F}$, and the $W_{\ell}$ obtained in the no-failure scenario), for all $\ell \in \mathcal{L}$. Finally, the procedure defines the capacity of the link $\ell$ as the maximum between them. Formally, we add a procedure $\operatorname{Max}()$, that performs this task. We symbolically write $W_{\ell}:=$ $\operatorname{Max}\left(W_{\ell}, \mathcal{W}_{1, \ell}, . . \mathcal{W}_{|\Omega|, \ell}\right)$. Together each final link capacity $W_{\ell}, \ell \in \mathcal{L}$ conform the final dimensioning set $\mathcal{W}$.

\section{NUMERICAL RESUlTS}

To quantify the quality of the CPLFT method, the proposed solution should be compared against the optimal solution. However, it is known that the RWAD problem is an NP-complete problem [34]. In fact, those who solved this problem optimally only have been able to achieve it to small networks (with less than 10 nodes). Consequently, for real network topologies (dozens to hundreds of nodes), the fault-tolerance problem cannot be optimally solved. Given this situation, our best alternative was to compare the CPLFT method with those methods considered as the most competitive at this moment.

Also, it is necessary to make a comparison using metrics that enable to assess the advantages/disadvantages of each fault tolerance method. The most important metrics for the Routing, Wavelength Dimensioning, and Fault Tolerance methods are the capacity of the network, and the delay in the restoration procedure in case of the occurrence of failures. In our approach this delay is comparable to the time needed to access the primary -or secondary- routes on routing tables since the CPLFT algorithm computations are executed offline (before the network operation), and the resulting primary and secondary routes are stored in tables. Next, we analyze which are the most suitable methods to be compared with. 


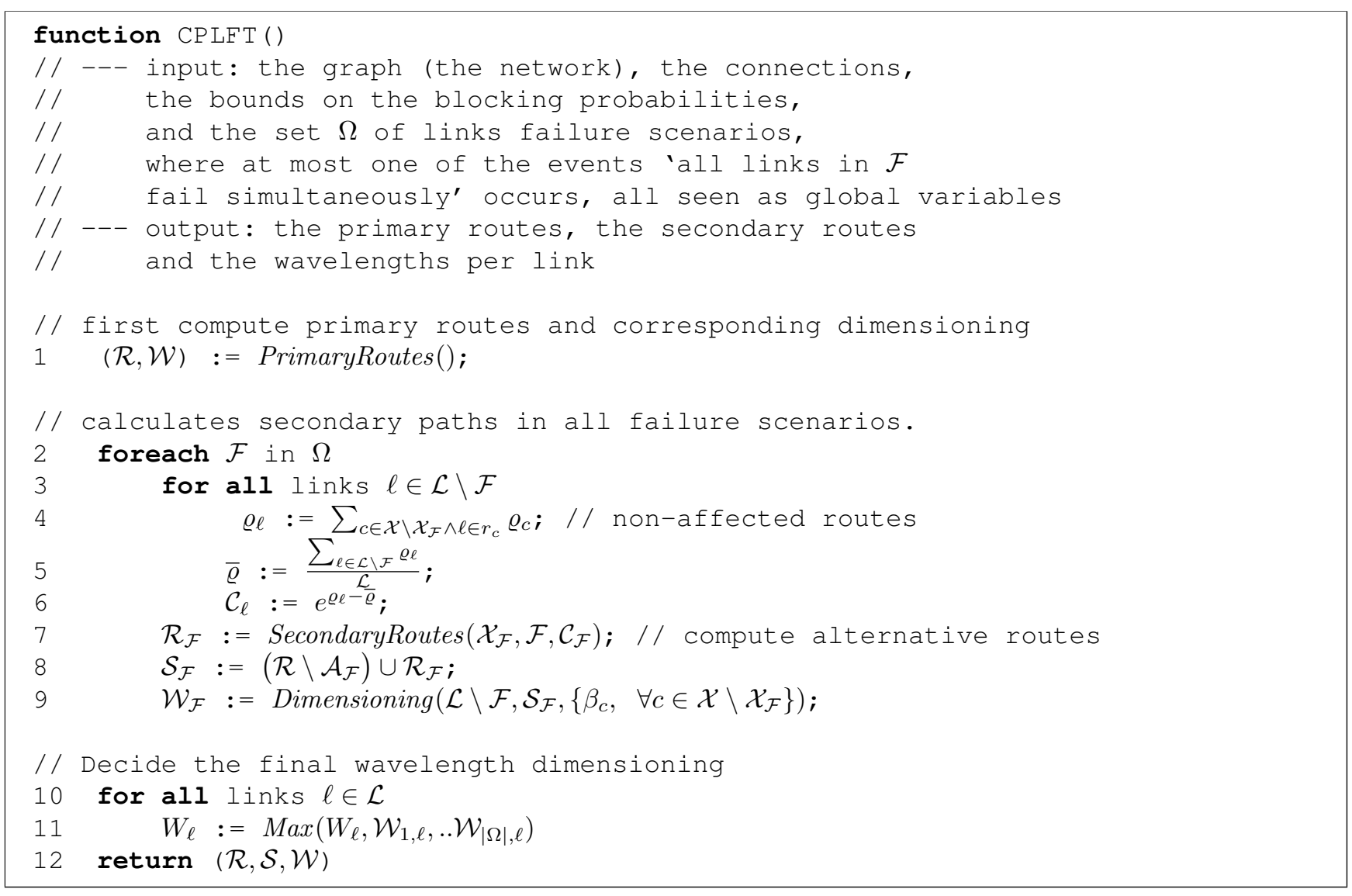

Figure 2. Algorithm for solving the RWAD problem, providing alternative routes if the links of one specific subset of links fail (all together) belonging to a list of possible subsets of links that can fail.
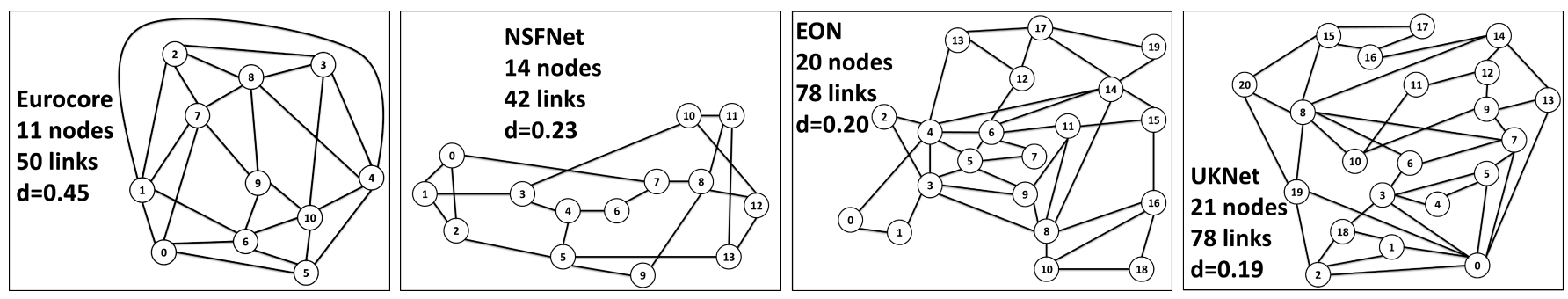

Figure 3. Mesh networks evaluated. The number of links refers to the number of bidirectional arcs. Observe that in the picture we see the edges (for instance, the picture shows the Eurocore topology with 25 edges, which corresponds to 50 arcs). The parameter $d$ is a measure of density: if the graph has $a$ arcs (twice the number of edges) and $n$ nodes, $d=a /(n(n-1))$.

a) Routing: Reviewing current methods of Routing, we notice that the algorithms most commonly referenced today, and considered the best so far, use the shortest path (both fixed and fixed alternate routing strategy), together with a First Fit wavelength assignment scheme. These are SP-FF (Shortest Path with First-Fit allocation scheme) and K-SPFF (K-disjoint routes for each user, it is possible to fulfill a transmission request if at least one of the $K$ routes is operational) [26], [27], [35]-[41].

It is interesting to note that the K-SP-FF technique is a fixed alternate routing method, which means that it uses several disjoint alternative routes to provide communication. By itself, it provides a certain level of fault tolerance, thanks to the alternative routes; however, it does not guarantee fault tolerance. This situation occurs since it is needed to define a restoration procedure together with a dimensioning procedure, which calculates the extra resources needed to guarantee fault-tolerance on diverse scenarios. Nevertheless, if we adjust the K-SP-FF to provide fault tolerance, satisfying the QoS restrictions, then the result would be the same as to add fault tolerance to the SP-FF method. Consequently, SP-FF was the routing method chosen for comparison.

b) Dimensioning: Additionally, the Wavelength Dimensioning method most commonly used nowadays is the dimensioning, that is, all links have the same amount of wavelengths. Consequently, in this work, we consider a 


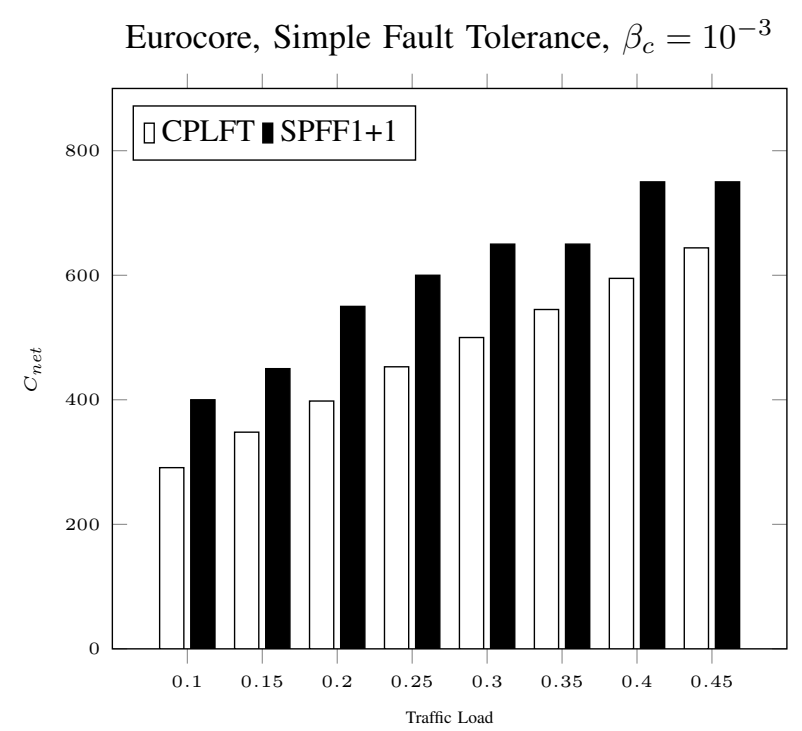

EON, Simple Fault Tolerance, $\beta_{c}=10^{-3}$

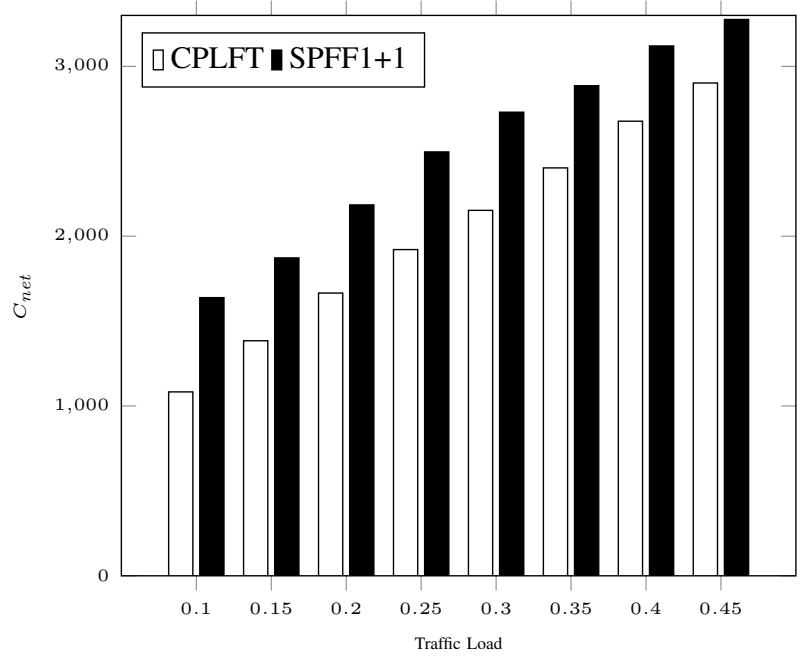

NSFNet, Simple Fault Tolerance, $\beta_{c}=10^{-3}$

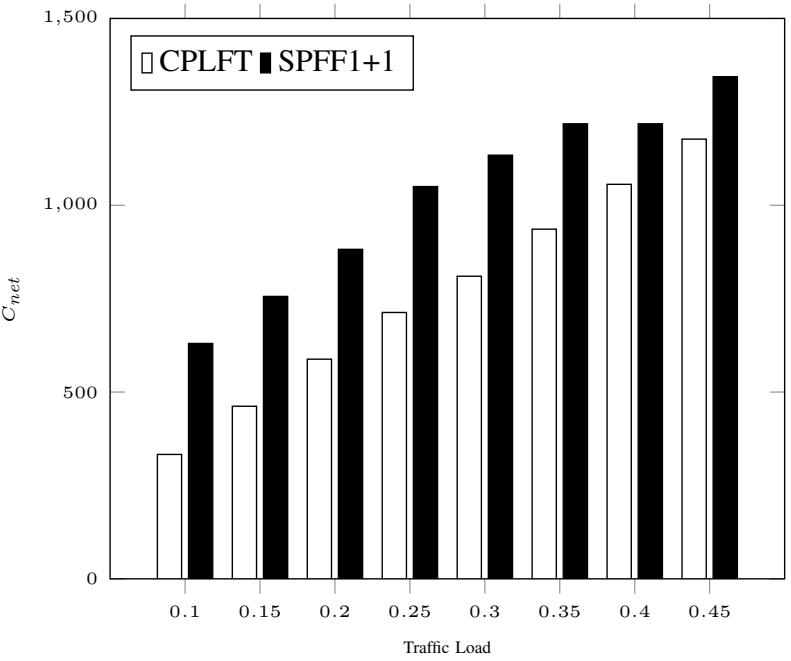

UKNet, Simple Fault Tolerance, $\beta_{c}=10^{-3}$

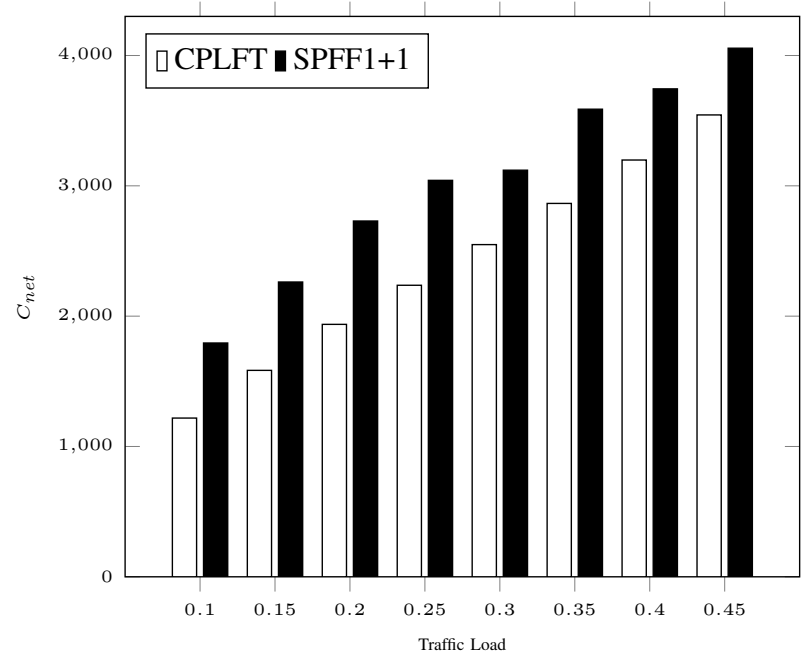

Figure 4. The total number of wavelengths $C_{n e t}$ obtained with our method (CPLFT) and SPFF1+1 on Eurocore, NSFNet, EON and UKNet real mesh network topologies, for different connection traffic loads with a blocking probability threshold $\beta_{c}$ equal to $10^{-3}$, in the single fault tolerance case.

homogeneous dimensioning strategy.

c) Fault Tolerance: As mentioned in the introduction, there are several types of fault tolerance algorithms proposed so far, such as Shared Path Protection, $p$-cycle and $1+1$. Hereafter, we discuss the pertinence in comparing CPLFT with each of these different types of algorithms.

Shared Path Protection (SPP) Method. As discussed in this paper introduction, this strategy provides tolerance to multiple network links failure. There are two methods for implementing this algorithm (on-line and off-line). Both methods require between 40 to $80 \%$ of additional wavelengths (compared to the case without fault tolerance) to provide single link fault tolerance capability [17]. Another aspect that must be considered is that the SPP off-line method has the additional weakness that the percentage of restoration obtained (percentage of connections that remain connected in case of link failure) is deficient (80\% to
90\% [17]), which means that it is not a fault-tolerant method. Therefore, it is not comparable to the method proposed in this work, which ensures that the blocking probability preestablished by the network designer (i.e. $10^{-6}$ ) is satisfied. On the other hand, the implementation of the SPP-online method requires to run on demand a route search algorithm (whenever one or more links fail) to find an alternative route to each affected connection. Evidently, this on-line strategy causes a slow re-routing, which added to the fact that many of the applications that use computer networks require swift on-line responses in case of failures [42], which implies that this type of method does not represent a practical fault-tolerant mechanism for many practical applications. Due to the facts just commented, the SPP method was not considered for comparison.

The p-cycle Method. As discussed early, to provide tolerance to multiple failures, this method requires a large number of 
Eurocore, Double Fault Tolerance, $\beta_{c}=10^{-3}$

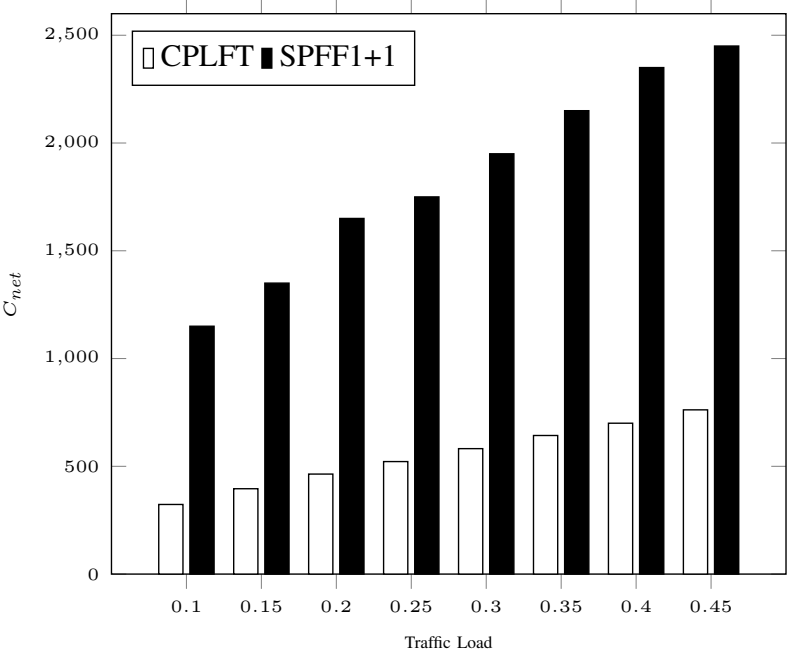

EON, Double Fault Tolerance, $\beta_{c}=10^{-3}$

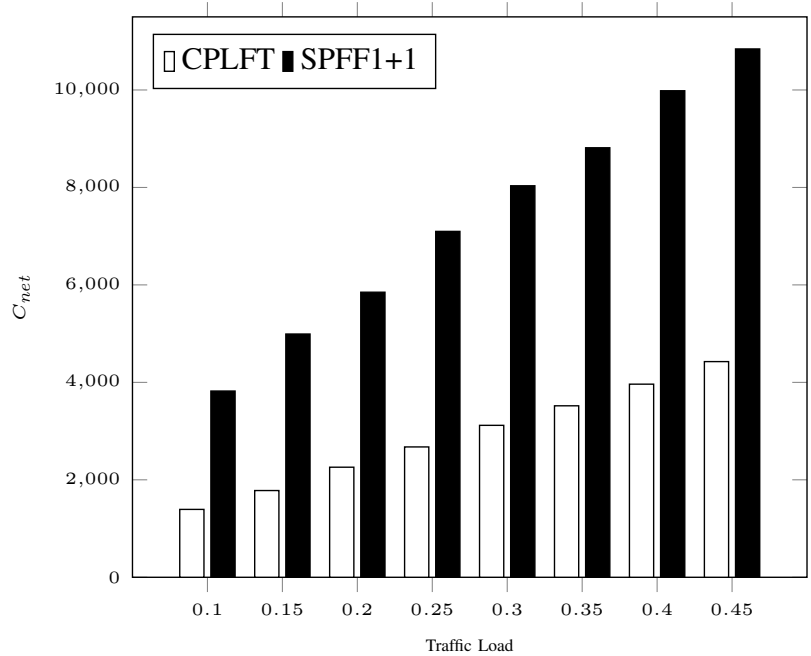

NSFNet, Double Fault Tolerance, $\beta_{c}=10^{-3}$

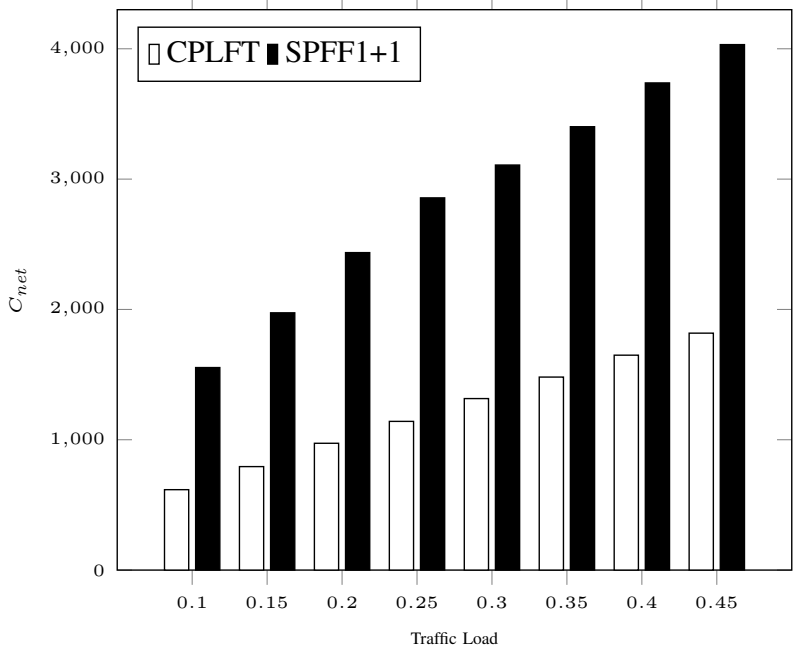

UKNet, Double Fault Tolerance, $\beta_{c}=10^{-3}$

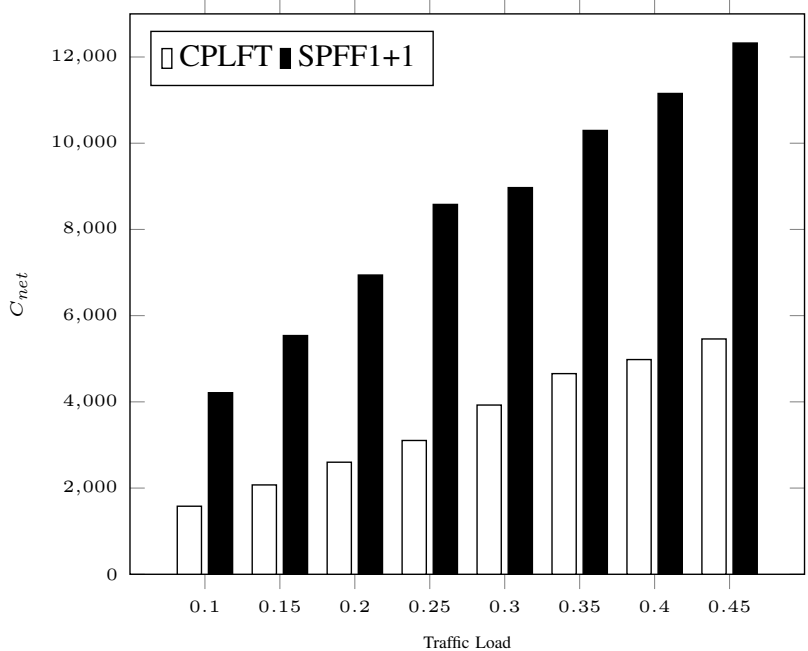

Figure 5. The total number of wavelengths $C_{n e t}$ obtained with our method (CPLFT) and SPFF1+1 on Eurocore, NSFNet, EON and UKNet real mesh network topologies, for different connection traffic loads with a blocking probability threshold $\beta_{c}$ equal to $10^{-3}$, in the simultaneous double fault tolerance case.

cycles (which implies a high cost when defining secondary routes), so it is not scalable for multiple faults. Given the fact that in this paper, we consider the multiple fault-tolerant cases, it is unreasonable to compare our method with the $p$ cycle one.

Method $1+1$. This method provides tolerance to multiple failures, using as many disjoint routes as simultaneous link failures considered. It solves the problem of primary and secondary routes before the network dimensioning (off-line) sub-task. Then, the number of wavelengths is computed, having as a constraint to provide enough resources to all routes, and providing sufficient information to re-route each connection in case of failure. Consequently, $1+1$ is the most suitable fault tolerance method to compare with our algorithm.

In summary, the most appropriate methods for comparison is: SP-FF to generate the primary routes; a homogeneous dimensioning strategy to compute the network capacity; and $1+1$ for the fault tolerance mechanism. These two methods together are denoted SPFF1+1 in the text. The mathematical method called LIBPE [33] was used to evaluate the corresponding blocking probabilities in both SPFF1+1 and CPLFT strategies, and the final results were validated by simulation.

To evaluate the performance of the methods under different scenarios, the algorithms were executed for different real network topologies, having different sizes and different degrees of connection $d$, where $d$ is the average number of neighbors of a node in the network. Some of the selected topologies and their respective parameters $N, L$ and $d$ are shown in Figure 3 .

Note that the total network capacity $C_{n e t}$ is the metric chosen to compare the algorithms, which is given by the total number of wavelengths necessary, to satisfy the users 
QoS constraints, including the primary and secondary routes of every connection $c$. Thus, in Figure 4 we show the total cost $C_{n e t}$ obtained by the CPLFT and SPFF1+1 methods for the case of a single link failure, as a function of the traffic load, for different network topologies, and a maximum acceptable blocking connection of $10^{-3}$. Additionally, Figure 5 shows the $C_{n e t}$ value for the same methods, but doublelink failures (i.e., any pair of simultaneous link failure possible). Notice that we show only single and double link failure scenarios. This is because to appropriately represent another kind of failures scenarios, such as SRG or Disaster Risk constraints may be hard to replicate and may achieve a disconnected network. However, as stated before, the algorithm developed can quickly evaluate any fault tolerance scenario.

As displayed in Figure 4 in the case of tolerance to a single failure, the CPLFT method performs better. In fact, for all the scenarios evaluated in our experiments, the SPFF1+1 method requires in general 30\% more wavelengths (for $\varrho=$ 0.3 , which is a representative network load [42]) than the cost of the method proposed herein.

In the case of tolerance to two simultaneous failures of links (Figure 5), the CPLFT method also significantly outperforms the SPFF $1+1$ technique. In this case, the SPFF $1+1$ method requires in the order of $160 \%$ more wavelengths (always for $\varrho=0.3[42]$ ) than our proposal.

Remark that for each scenario analyzed herein, both compared methods achieve to connect the same users (connections) with the same QoS requirements (maximum acceptable blocking probability), but our proposal requires significantly fewer resources than SPFF1+1 to do so.

\section{COnclusions}

A novel method was proposed to jointly solve an important problem in designing dynamic optical network with wavelength continuity constraint. It determines the set of primary and the set of secondary routes, using a fixed routing strategy. Also, it evaluates the minimum number of wavelengths necessary on each network link so that the blocking probability of each user is lower than a given pre-specified threshold (which is a design parameter of the network). Additionally, it solves the fault-tolerance problem for any possible set of scenarios, where each scenario is defined by a specific set of link failures.

In general, the method differs considerably from those published so far, obtaining better results in terms of the necessary number of wavelengths and delay. Additionally, the dimensioning method does not make any distinction between primary and alternative routes, with the constraint that it only evaluates scenarios that may happen during the network operation (for each user, it considers either a primary or a secondary route, not both simultaneously). The method allows sharing the resources between the secondary routes while guaranteeing a maximum blocking probability to each network connection.

The fault tolerance technique is scalable to any set of simultaneous link failures, as long as the network topology allows reconnection via the links that remain operational. This scheme is executed before the network operation, requiring a few seconds to solve the task. This fast execution also allows to quickly solve any link failure scenario in case of variations during network operation (for example, traffic load variations). Additionally, the network operation based on our approach is fast and straightforward, since the routes (both primary and secondary) are stored in routing tables and consulted only on demand.

\section{ACKNOWLEDGMENTS}

This work received financial support from FONDEF ID14I2012, CONICYT and STICAMSUD 19STIC-01. These projects are then gratefully acknowledged.

\section{REFERENCES}

[1] M. To and P. Neusy, "Unavailability analysis of long-haul networks," Selected Areas in Communications, IEEE Journal on, vol. 12, no. 1, pp. 100-109, 1994.

[2] D. A. Schupke, A. Autenrieth, and T. Fischer, "Survivability of Multiple Fiber Duct Failures," in Third International Workshop on the Design of Reliable Communication Networks (DRCN, pp. 7-10, 2001.

[3] R. Ramaswami and K. N. Sivarajan, "Design of logical topologies for wavelength-routed all-optical networks,' in INFOCOM '95. Fourteenth Annual Joint Conference of the IEEE Computer and Communications Societies. Bringing Information to People. Proceedings. IEEE, pp. 1316-1325 vol.3, 1995.

[4] H. Singh, J. Prakash, D. Arora, and A. Wason, "Fault Tolerant Congestion Based Algorithms in $\{\mathrm{O}\}\{\mathrm{B}\}\{\mathrm{S}\}$ Network," International Journal of Engineering (IJE), vol. 5, no. 5, 2011.

[5] F. S. H. Souza, D. L. Guidoni, and G. R. Mateus, "A column generation-based heuristic for the GRWA with protection and QoS in WDM optical networks," in Computers and Communications (ISCC), 2013 IEEE Symposium on, pp. 922-927, 2013.

[6] D. S. Mukherjee, C. Assi, and A. Agarwal, "Alternate Strategies for Dual Failure Restoration Using p-Cycles," in Communications, 2006. ICC '06. IEEE International Conference on, vol. 6, pp. 2477-2482, 2006.

[7] R. Yadav, R. Yadav, and H. Singh, "Intercycle switching (ICS)-based dynamic reconfiguration of p-cycle for dual-failure survivability of WDM networks," Photonic Network Communications, vol. 24, no. 2, pp. 160-165, 2012.

[8] D. S. Yadav, S. Rana, and S. Prakash, "A mixed connection recovery strategy for surviving dual link failure in $\{$ WDM $\}$ networks," Optical Fiber Technology, vol. 19, no. 2, pp. 154-161, 2013.

[9] M. Jinno, T. Takagi, and Y. Uemura, "Enhanced survivability of translucent elastic optical network employing shared protection with fallback," in 2017 Optical Fiber Communications Conference and Exhibition (OFC), pp. 1-3, March 2017.

[10] D. Serre and C. Heinzlef, "Assessing and mapping urban resilience to floods with respect to cascading effects through critical infrastructure networks," International Journal of Disaster Risk Reduction, 2018.

[11] F. Dikbiyik, M. Tornatore, and B. Mukherjee, "Minimizing the risk from disaster failures in optical backbone networks," Journal of Lightwave Technology, 2014.

[12] S. Ferdousi, F. Dikbiyik, M. F. Habib, M. Tornatore, and B. Mukherjee, "Disaster-aware dynamic content placement in optical cloud networks," in Conference on Optical Fiber Communication, Technical Digest Series, 2014.

[13] X. Shao, Y. Bai, X. Cheng, Y.-K. Yeo, L. Zhou, and L. H. Ngoh, "Best Effort SRLG Failure Protection for Optical WDM Networks," Journal of Optical Communications and Networking, 2011.

[14] P. Babarczi, J. Tapolcai, P. H. Ho, and M. Medard, "Optimal dedicated protection approach to shared risk link group failures using network coding," in IEEE International Conference on Communications, 2012.

[15] S. Ramamurthy, L. Sahasrabuddhe, and B. Mukherjee, "Survivable WDM mesh networks," Lightwave Technology, Journal of, vol. 21, no. 4, pp. 870-883, 2003. 
[16] M. Wang, S. Li, E. W. M. Wong, and M. Zukerman, "Performance Analysis of Circuit Switched Multi-Service Multi-Rate Networks With Alternative Routing," Journal of Lightwave Technology, vol. 32, no. 2 , pp. 179-200, 2014.

[17] D. Schupke and R. Prinz, "Capacity Efficiency and Restorability of Path Protection and Rerouting in WDM Networks Subject to Dual Failures," Photonic Network Communications, vol. 8, no. 2, pp. 191207, 2004.

[18] A. Wason and R. S. Kaler, "Fault-tolerant routing and wavelength assignment algorithm for multiple link failures in wavelength-routed all-optical $\{$ WDM $\}$ networks," Optik - International Journal for Light and Electron Optics, vol. 122, no. 2, pp. 110-113, 2011.

[19] D. Pereira and M. Camillo Penna, "A new algorithm for dimensioning resilient optical networks for shared-mesh protection against multiple link failures," Optical Switching and Networking, 2014.

[20] D. A. Schupke, "Multiple failure survivability in WDM networks with p-cycles," in Circuits and Systems, 2003. ISCAS '03. Proceedings of the 2003 International Symposium on, vol. 3, pp. III-866-III-869 vol.3, may 2003.

[21] L. Tang, M. Cai, B. Li, and R. Wu, "A novel multi-link faulttolerant algorithm for survivability in multi-domain optical networks," Photonic Network Communications, vol. 24, no. 2, pp. 77-85, 2012.

[22] F. Ji, X. Chen, W. Lu, J. J. Rodrigues, and Z. Zhu, "Dynamic pcycle configuration in spectrum-sliced elastic optical networks," in GLOBECOM - IEEE Global Telecommunications Conference, 2013.

[23] W. Liao and C. H. Loi, "Providing service differentiation for opticalburst-switched networks," Journal of Lightwave Technology, vol. 22, no. 7, pp. 1651-1660, 2004

[24] D. H. Hailu, G. G. Lema, E. A. Yekun, and S. H. Kebede, "Unified study of Quality of Service (QoS) in OPS/OBS networks," Optical Fiber Technology, vol. 36, pp. 394-402, jul 2017.

[25] S. Mohd Sam, S. Mohd Daud, K. Kamardin, and N. Maarop, "Study of Qos Performance in Optical Burst Switched Networks (OBS)," Indian Journal of Science and Technology, vol. 9, dec 2016.

[26] A. Zapata-Beghelli and P. Bayvel, "Dynamic Versus Static Wavelength-Routed Optical Networks," Lightwave Technology, Journal of, vol. 26, pp. 3403-3415, oct 2008.

[27] R. Ramaswami, K. Sivarajan, and G. Sasaki, Optical Networks: A Practical Perspective, 3rd Edition. San Francisco, CA, USA: Morgan Kaufmann Publishers Inc., 3rd ed., 2009.

[28] N. Jara, R. Vallejos, and G. Rubino, "A method for joint routing, wavelength dimensioning and fault tolerance for any set of simultaneous failures on dynamic WDM optical networks," Optical Fiber Technology, vol. 38, pp. 30-40, 2017.

[29] F. Dikbiyik, A. S. Reaz, M. De Leenheer, and B. Mukherjee, "Minimizing the disaster risk in optical telecom networks," in Optical Fiber Communication Conference, pp. OTh4B--2, Optical Society of America, 2012.

[30] S. Ferdousi, F. Dikhiyik, M. F. Habib, and B. Mukherjee, "Disasteraware data-center and content placement in cloud networks," in 2013 IEEE International Conference on Advanced Networks and Telecommunications Systems (ANTS), pp. 1-3, IEEE, 2013.

[31] H. Zang, C. Ou, and B. Mukherjee, "Path-protection routing and wavelength assignment in WDM mesh networks under shared-risk-group constraints," in Asia-Pacific Optical and Wireless Communications Conference and Exhibit, pp. 49-60, International Society for Optics and Photonics, 2001

[32] E. W. Dijkstra, "A note on two problems in connexion with graphs," Numerische Mathematik, vol. 1, no. 1, pp. 269-271, 1959.

[33] N. Jara, R. Vallejos, and G. Rubino, "Blocking Evaluation and Wavelength Dimensioning of Dynamic WDM Networks Without Wavelength Conversion," Journal of Optical Communications and Networking, vol. 9, no. 8, p. 625, 2017.

[34] R. Vallejos, A. Zapata-Beghelli, V. Albornoz, and M. Tarifeño, "Joint routing and dimensioning of optical burst switching networks," Photonic Network Communications, vol. 17, no. 3, pp. 266-276, 2009.

[35] I. Chlamtac, A. Ganz, and G. Karmi, "Lightpath communications: an approach to high bandwidth optical WAN's," Communications, IEEE Transactions on, vol. 40, pp. 1171-1182, jul 1992.

[36] H. Zang, J. P. Jue, B. Mukherjee, and Others, "A review of routing and wavelength assignment approaches for wavelength-routed optical $\{\mathrm{W}\}\{\mathrm{D}\}\{\mathrm{M}\}$ networks," Optical Networks Magazine, vol. 1, no. 1, pp. 47-60, 2000
[37] J. Teng and G. Rouskas, "Traffic engineering approach to path selection in optical burst switching networks," J. Opt. Netw., vol. 4, pp. 759-777, nov 2005 .

[38] J. Zheng, B. Zhang, and H. T. Mouftah, "Toward automated provisioning of advance reservation service in next-generation optical Internet," IEEE Communications Magazine, vol. 44, no. 12, pp. 68-74, 2006.

[39] N. Charbonneau and V. M. Vokkarane, "A survey of advance reservation routing and wavelength assignment in wavelength-routed WDM networks," IEEE Communications Surveys and Tutorials, vol. 14, no. 4, pp. 1037-1064, 2012.

[40] B. C. Chatterjee, N. Sarma, and P. Pratim Sahu, "Review and Performance Analysis on Routing and Wavelength Assignment Approaches for Optical Networks.," IETE Technical Review, vol. 30, no. 1, pp. 12 23, 2013.

[41] R. T. Koganti and D. Sidhu, "Analysis of routing and wavelength assignment in large WDM networks," in Procedia Computer Science, vol. 34, pp. 71-78, 2014.

[42] A. A. M. Saleh and J. M. Simmons, "Technology and architecture to enable the explosive growth of the internet," Communications Magazine, IEEE, vol. 49, no. 1, pp. 126-132, 2011. 